\title{
Endoscopic regression of Barrett's oesophagus during omeprazole treatment; a randomised double blind study
}

\author{
F T M Peters, S Ganesh, E J Kuipers, W J Sluiter, E C Klinkenberg-Knol,
} C B H W Lamers, J H Kleibeuker

\begin{abstract}
Background-Barrett's oesophagus, columnar metaplasia of the epithelium, is a premalignant condition with a 50-100-fold increased risk of cancer. The condition is caused by chronic gastro-oesophageal reflux. Regression of metaplasia may decrease the cancer risk.

Aims-To determine whether elimination of acid gastro-oesophageal reflux induces a regression of metaplastic epithelium. Methods-Sixty eight patients with acid reflux and proven Barrett's oesophagus were included in a prospective, randomised, double blind study with parallel groups, and were treated with profound acid secretion suppression with omeprazole $40 \mathrm{mg}$ twice daily, or with mild acid secretion suppression with ranitidine 150 mg twice daily, for 24 months. Endoscopy was performed at $0,3,9,15$, and 24 months with measurement of length and surface area of Barrett's oesophagus; pH-metry was performed at 0 and 3 months. Per protocol analysis was performed on 26 patients treated with omeprazole, and 27 patients treated with ranitidine.
\end{abstract}

Results-Omeprazole reduced reflux to $0.1 \%$, ranitidine to $9.4 \%$ per 24 hours. Symptoms were ameliorated in both groups. There was a small, but statistically significant regression of Barrett's oesophagus in the omeprazole group, both in length and in area. No change was observed in the ranitidine group. The difference between the regression in the omeprazole and ranitidine group was statistically significant for the area of Bartrend in the same direction for the length of Barrett's oesophagus $(\mathrm{p}=0.06)$.

Conclusions-Profound suppression of acid secretion, leading to elimination of acid reflux, induces partial regression of Barrett's oesophagus.

(Gut 1999;45:489-494)

Keywords: acid reflux; Barrett's oesophagus; omeprazole; regression

Correspondence to: Dr F T M Peters, University Hospital Groningen,

Department of

Gastroenterology, PO Box

30.001, 9700 RB Groningen,

The Netherlands.

Accepted for publication 27 April 1999 Barrett's oesophagus is a condition in which squamous epithelium of the oesophagus has been replaced by metaplastic columnar epithelium to a variable extent. ${ }^{1}$ Barrett's oesophagus is a premalignant condition, with a 50-100fold increased risk for oesophageal cancer, rett's oesophagus $(p=0.02)$, and showed a

compared with the risk in the general population. ${ }^{2-6}$ In the large majority of patients Barrett's oesophagus is due to chronic acid gastro-oesophageal reflux. Reflux reducing treatment - that is, treatment with acid inhibitory drugs, generally relieves symptoms adequately, and when given in sufficient doses, heals concomitant oesophagitis and Barrett's ulcers and helps to prevent restenosis after instrumental dilatation. Whether such treatment may also modify the risk of cancer development is however unknown. Although dysplasia is an important, if not the most important marker for cancer risk, the length of Barrett's epithelium also seems to be a risk factor for dysplasia or adenocarcinoma. ${ }^{3}$ Thus a decrease of cancer risk might be anticipated if treatment could induce a regression of Barrett's epithelium with restitution of squamous epithelium. A number of case reports ${ }^{89}$ and non-randomised, open label studies ${ }^{10-12}$ have indeed suggested that regression of Barrett's oesophagus can be accomplished by antireflux treatment. However, other studies could not confirm this. ${ }^{13-15}$ To clarify this issue we performed a prospective, double blind, randomised study comparing the effect of elimination of acid reflux with omeprazole with the effect of reduction of acid reflux with conventional dose ranitidine, sufficient for acceptable symptom relief. The primary end points of this study were the change in length and total area of Barrett's oesophagus during a two year treatment course.

\section{Patients and methods}

PATIENTS

Patients with known Barrett's oesophagus and newly diagnosed patients in the participating hospitals took part in the study. They were eligible if they fulfilled the following criteria: (1) endoscopically and histologically proven Barrett's oesophagus ${ }^{1}$ over a distance of at least $3 \mathrm{~cm}$ from the endoscopically determined oesophagogastric junction; (2) documented acid gastro-oesophageal reflux, defined as eosophageal $\mathrm{pH}<4$ for more than $1 \%$ of a 24 hour period while using low dose ranitidine (not more than $150 \mathrm{mg}$ twice daily), or no antireflux medication at all; and (3) age between 18 and 75 years.

Exclusion criteria were: (1) benign oesopha-
Abbreviations used in this paper: GORD, gastro-oesophageal reflux disease; AUC, area under the curve; CI, confidence interval. 


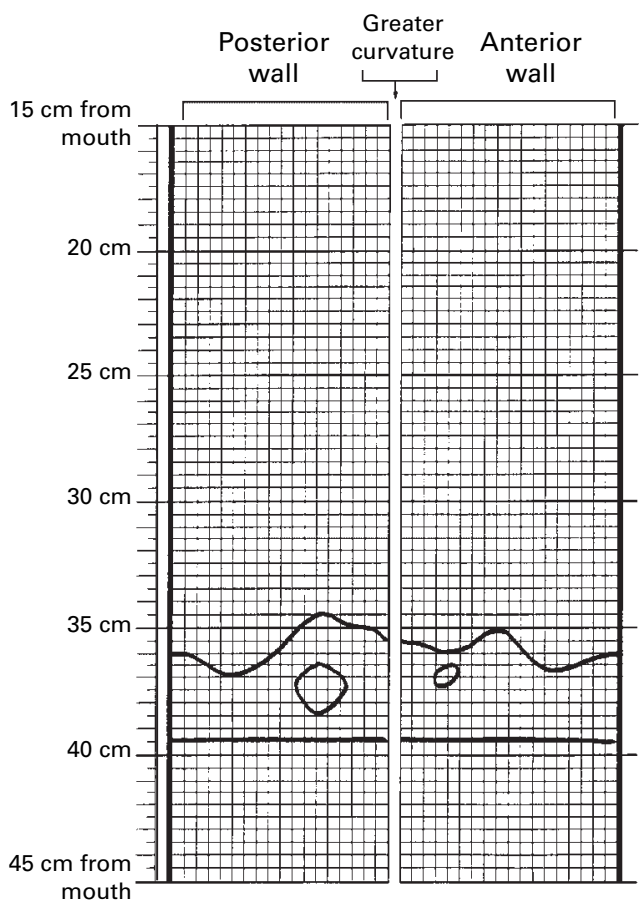

Figure 1 Example of a drawing, sketched on graph paper, with the squamocolumnar junction, two squamous islands within Barrett's epithelium, and the oesophagogastric junction.

repeated endoscopy; (2) oesophageal carcinoma or high grade dysplasia; (3) the requirement for acid suppressing medication stronger than ranitidine $150 \mathrm{mg}$ twice daily or its equivalent for symptomatic relief, or the requirement for ranitidine $150 \mathrm{mg}$ twice daily or its equivalent, in combination with prokinetic drugs stronger than the equivalent of cisapride $10 \mathrm{mg}$ four times daily; and (4) contraindications to the use of omeprazole or ranitidine: pregnancy or lactation; haemorrhagic diathesis or the use of antithrombotic drugs if the dosing could not be adjusted prior to endoscopy; or concurrent therapy with cytotoxic drugs. The study was approved by the Medical Ethical Committees of the participating hospitals and each patient gave either witnessed verbal or written informed consent.

STUDY PROTOCOL

After the initial examinations (see later) patients were allocated to treatment with either omeprazole $40 \mathrm{mg}$ twice daily or ranitidine 150 mg twice daily (as a control group) according to a computer generated randomisation list. The randomisation was made separately for each centre and in blocks of two consecutive patients. The study was rendered double blind using the double dummy technique. The drugs were given for a total duration of two years. Endoscopy was performed at baseline and after $3,9,15$, and 24 months, while on medication. Patients were seen every three months, at which time study medication was collected for determination of patient compliance and new medication was provided.

CLINICAL PARAMETERS

At baseline and every three months thereafter patients were questioned about their reflux symptoms-heartburn, regurgitation, dysphagia, and odynophagia. The symptoms were graded from 0 to 3 ( $0=$ none; $1=$ mild, occasional; $2=$ moderate, occasionally interfering with normal acitvities, predictable from position, requiring liquids to clear; $3=$ severe, constant interference of heartburn with normal activities, pulmonary aspiration, episodes of oesophageal obstruction). Adverse events were noted.

ENDOSCOPY AND MEASUREMENT OF LENGTH AND TOTAL AREA OF BARRETT'S OESOPHAGUS In each of the three centres the great majority of endoscopies were performed by one experienced endoscopist, with one other endoscopist as back up in case of unforeseen circumstances. Endoscopy was performed with standard forward viewing endoscopes. During the procedure the diaphragmatic pinch, the oesophagogastric junction, and the squamocolumnar junction were localised on slow withdrawal of the endoscope. The oesophagogastric junction was defined to be at the upper border of the gastric folds as viewed after desufflation. For the measurement of the length of Barrett's oesophagus the highest point of the squamocolumnar junction was taken. All distances were measured in relation to the incisors.

In order to determine the total area of Barrett's mucosa, the endoscopist made a drawing on graph paper of the mucosal landmarks, the course of the $\mathrm{Z}$ line, and of islands of squamous and metaplastic epithelium during or immediately after each endoscopy. The area of Barrett's mucosa was expressed as the number of complete squares within the borders of the metaplastic epithelium, including islands of metaplastic epithelium proximal to the $\mathrm{Z}$ line and excluding islands of squamous epithelium distal to the $Z$ line (fig 1). Intraobserver coefficient of variability for both the measuring of length and of area of Barrett's oesophagus was calculated from the paired data at baseline and at three months in the ranitidine group.

Apart from the above mentioned parameters the oesophagitis was scored from grade 0 to 4 $(0=$ normal; 1 = round or linear erythematous spots, with or without fibrinous coat; $2=$ isolated round or linear erosions extending from the gastro-oesophageal junction upwards, non-circumferential; $3=$ confluent erosions extending around the entire circumference of superficial ulceration, without stenosis; $4=$ complicated, erosions as described plus deep ulceration, stricture).

\section{pH-METRY}

Ambulatory 24 hour oesophageal pH-metry was performed at baseline, while the patient took no acid reducing drugs or an $\mathrm{H}_{2}$ receptor antagonist in a dose not exceeding the equivalent of ranitidine $150 \mathrm{mg}$ twice daily. pH-metry was repeated three months after the start of the study while the patient was on study medication. The endoscopists were not aware of the results of the pH-metry at the subsequent endoscopies. The $\mathrm{pH}$-metries were performed using glass electrodes (Ingold, 
Table 1 Patient characteristics at $t=0$

\begin{tabular}{llll}
\hline & Omeprazole & Ranitidine & p Value \\
\hline Number & 31 & 30 & \\
Age (years) & $58.0(53.5-62.0)$ & $56.0(51.0-60.5)$ & 0.63 \\
Sex (M/F) & $23 / 8$ & $20 / 10$ & 0.72 \\
Length of Barrett's oesophagus (cm) & $5.6(5.0-7.0)$ & $5.0(4.5-6.5)$ & 0.68 \\
Surface area of Barrett's oesophagus (squares) & $406(345-487)$ & $367(300-466)$ & 0.55 \\
Reflux & & & \\
$\quad$ Total (\%) & $19.7(12.3-32.4)$ & $14.3(11.3-18.2)$ & 0.38 \\
Erect (\%) & $16.5(10.6-30.0)$ & $13.7(9.4-18.1)$ & 0.60 \\
Supine (\%) & $21.1(13.1-39.2)$ & $13.0(8.4-18.4)$ & 0.10 \\
LOSP (mm Hg) & $7(5-9)$ & $8(5-10)$ & 0.51 \\
Oesophagitis (grade 0/1/2/3/4) & $17 / 7 / 6 / 1 / 0$ & $23 / 2 / 3 / 2 / 0$ & $>0.15^{\star}$ \\
Symptoms (grade 0/1/2/3) & $6 / 13 / 12 / 0$ & $5 / 17 / 8 / 0$ & $>0.40^{\star}$ \\
$\quad$ Heartburn & $12 / 12 / 7 / 0$ & $12 / 12 / 6 / 0$ & $>0.95^{\star}$ \\
Regurgitation & $29 / 2 / 0 / 0$ & $25 / 5 / 0 / 0$ & $0.40^{\star}$ \\
Dysphagia & $24 / 5 / 1 / 1$ & $26 / 4 / 0 / 0$ & $0.55^{\star}$ \\
$\quad$ Odynophagia & & & \\
\hline
\end{tabular}

LOSP, lower oesophageal sphincter pressure.

Results expressed as mean values ( $95 \%$ confidence interval), non-parametric. ${ }^{\star} \chi^{2}$ test. changes within a group, and Mann-Whitney test for differences between the groups). Changes in score within a group were analysed by the test of Pitman, whereas differences in scores and in changes of scores between the groups were analysed by the test of Yates and Cochran. Data are given as the statistical means and their 95\% confidence intervals (CI). Differences in frequencies were studied by $\chi^{2}$ analysis.

\section{Results}

Sixty eight patients entered the study. At baseline, 46 were on a maintenance dose of $\mathrm{H}_{2}$ blocking drugs equivalent to ranitidine $150 \mathrm{mg}$ twice daily, and 17 used $\mathrm{H}_{2}$ blockers occasionally or not at all. After inclusion five patients proved to use a higher dose of $\mathrm{H}_{2}$ blockers than allowed according to the protocol. As these five still had the required acid reflux, they were not withdrawn from the study. Of the 68 patients, 33 were assigned to omeprazole and 35 to ranitidine.

Of the patients assigned to omeprazole, one never took his study drug; for one patient the treatment code was broken because of persistent substernal complaints; four patients were withdrawn because of adverse events (one with oesophageal adenocarcinoma, and one with myocardial infarction with no apparent causal relation to the study drug; one with diarrhoea plus arthralgia, and one with complaints of swollen eyelids, itching, tears, and blurred vision, in both assessed as probably caused by the study drug); one patient dropped out because of personal reasons.

Of the patients assigned to ranitidine, one patient was withdrawn because of pregnancy, one patient because of an adverse event (malignant pleural effusion, primary tumour unknown); in six medication was changed into open label omeprazole treatment because of insufficient gastro-oesophageal reflux disease (GORD) symptom reduction.

Of the original 68 randomised patients, 26 in the omeprazole group and 27 in the ranitidine group thus completed the study with the assigned drug and were suitable for statistical analysis at 24 months.

All but three patients had intestinal metaplasia evident in at least one biopsy specimen; $75 \%$ of the patients revealed no dysplasia at inclusion, and $25 \%$ had indefinite/low grade dysplasia.The omeprazole and patients on ranitidine were comparable for age, sex, length of Barrett's oesophagus, and degree of reflux, as well as for their grade of macroscopic oesophagitis and their symptom score (table 1). This was equally true for the initially randomised patients, including the nonstarters and those who dropped out early, and for those who completed the study (latter data not shown). Compliance to the study medication was more than $90 \%$ in both groups.

Omeprazole $40 \mathrm{mg}$ twice daily almost completely eliminated acid reflux. Ranitidine $150 \mathrm{mg}$ twice daily induced a statistically significant decrease of acid reflux, but reflux remained pathologically increased (table 2). Symptom reduction was modest in both
Results expressed as mean values (95\% confidence interval), non-parametric.

${ }^{\star}$ Omeprazole $v$ ranitidine. 
Table 3 Change in symptom score (scale 0-3), between 0 and 24 months

\begin{tabular}{llll}
\hline & Omeprazole & Ranitidine & p Valuet \\
\hline Study completed $(\mathrm{n})$ & 26 & 27 & \\
Heartburn & $-1.08^{\star}(-1.65$ to -0.51$)$ & $-0.67^{\star}(-1.10$ to -0.23$)$ & 0.13 \\
Regurgitation & $-0.50^{\star}(-0.90$ to -0.10$)$ & $-0.70^{\star}(-1.16$ to -0.25$)$ & 0.48 \\
Dysphagia & $0.0(-0.15$ to +0.15$)$ & $-0.11(-0.31$ to +0.09$)$ & 0.30 \\
Odynophagia & $-0.19(-0.43$ to +0.05$)$ & $-0.11(-0.31$ to +0.09$)$ & 0.73 \\
\hline
\end{tabular}

Results expressed as mean values (95\% confidence interval), non-parametric.

* Statistically significant according to Pitman.

†Omeprazole $v$ ranitidine; according to Yates and Cochran.

Table 4 Change in length (absolute and relative) of Barrett's oesophagus, expressed as the area under the curve (AUC), derived from measurements at 0, 3, 9, 15, and 24 months, compared with no change

\begin{tabular}{llll}
\hline & Omeprazole $(n=26)$ & Ranitidine $(n=27)$ & $p$ Valuef \\
\hline $\begin{array}{l}\text { AUC_change in length (cm/month) } \\
\text { AUC_relative change in length } \\
(\% / \text { month })\end{array}$ & $-6.4^{\star}(-15.8$ to -0.8$)$ & $-0.0(-4.5$ to +4.5$)$ & 0.06 \\
\hline
\end{tabular}

Results expressed as mean values (95\% confidence interval).

* Statistically significant, non-parametric paired Wilcoxon.

†Omeprazole $v$ ranitidine; Mann-Whitney U test.

Table 5 Change in surface area (absolute and relative) of Barrett's oesophagus, expressed as the area under the curve (AUC), derived from measurements at 0,3,9, 15, and 24 months, compared with no change

\begin{tabular}{llll}
\hline & Omeprazole $(n=26)$ & Ranitidine $(n=27)$ & $p$ Valuet \\
\hline $\begin{array}{l}\text { AUC_change in area (squares/month) } \\
\text { AUC_relative change in area }\end{array}$ & $-862^{\star}(-1466$ to -302$)$ & $-11(-312$ to +236$)$ & 0.02 \\
$(\% /$ month $)$ & $-9.0^{\star}(-15.0$ to -3.0$)$ & $+0.1(-3.6$ to +2.8$)$ & 0.02 \\
\hline
\end{tabular}

Results expressed as mean values (95\% confidence interval).

* Statistically significant, non-parametric paired Wilcoxon.

†Omeprazole $v$ ranitidine; Mann-Whitney U test.

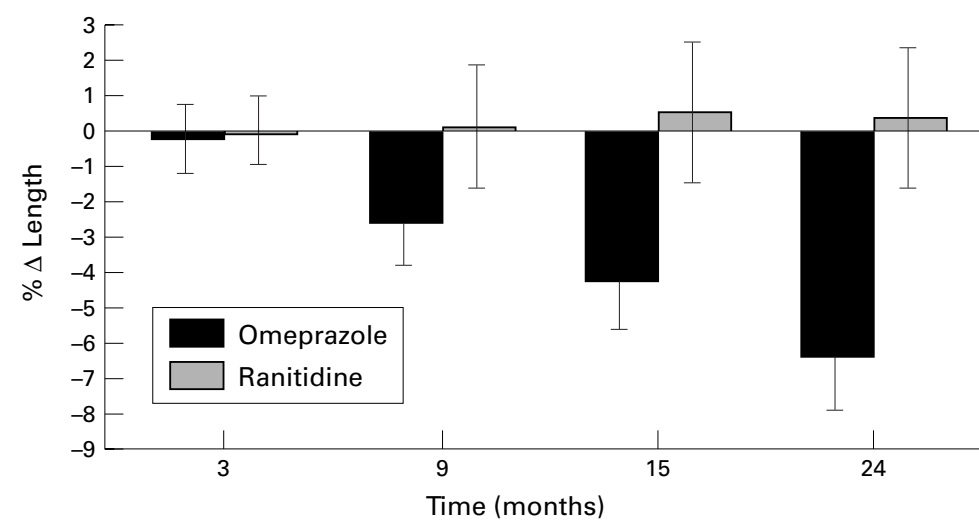

Figure 2 Relative changes in length of Barrett's oesophagus in the omeprazole and ranitidine treated patients (mean values (SEM)) at 3, 9, 15 and 24 months, compared with baseline.

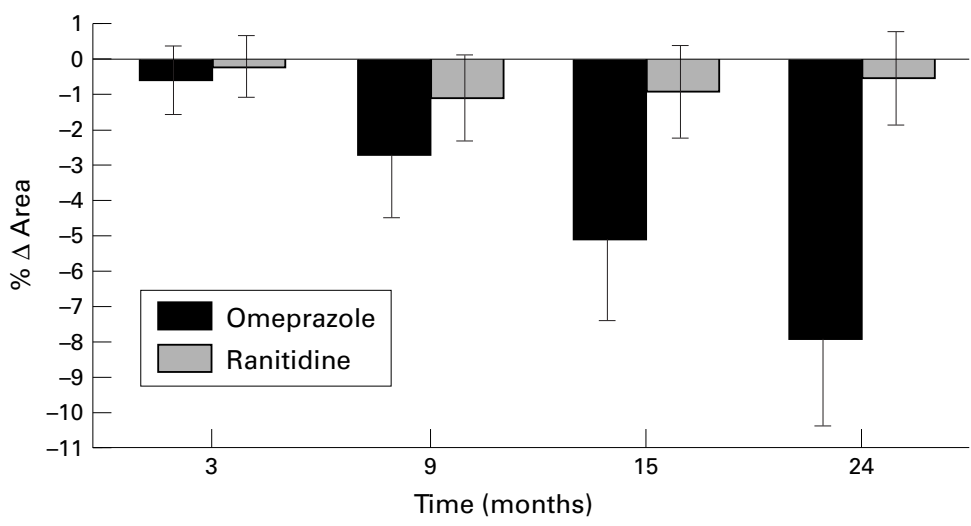

Figure 3 Relative changes in surface area of Barrett's oesophagus in the omeprazole and ranitidine treated patients (mean values (SEM)) at 3, 9, 15, and 24 months, compared with baseline. groups, which may have been partly due to a low symptom score at the start of the study. There was no statistically significant difference between the two drugs with respect to the clinical parameters (table 3). Of those who had endoscopically active oesophagitis at the start, oesophagitis improved in 10 patients on omeprazole and in six patients on ranitidine; the oesophagitis worsened in none of the patients on omeprazole and in three of the patients on ranitidine. In the majority the score remained low and did not change. These differences in macroscopic change in oesophagitis, however, did not reach statistical significance.

Intraobserver coefficient of variability was $5 \%$ for measurement of the length, and $4.5 \%$ for the area of Barrett's oesophagus. Elimination of acid reflux by omeprazole resulted in a significant reduction of both the length (table 4) and the total area (table 5) of Barrett's oesophagus, whereas no change was noted during persistent reflux in the ranitidine group. When the results in the omeprazole group and the ranitidine group were compared, a statistically significant difference was found between the effects on total area of Barrett's oesophagus (table 5); the difference between the effects on length showed a trend in the same direction (table 4). When only the lengths and the areas as measured at baseline and after two years were compared with each other, the changes in both length and area in the omeprazole group were significant $(-6.4 \%$ (CI -3.2 to $-9.5 \%)$ and $-7.9 \%$ (CI -3.0 to $-12.9 \%$ ), respectively), but in the ranitidine group they were not $(0.4 \%$ (CI 4.3 to $-3.5 \%$ ) and $-0.6 \%$ (CI $3.3 \%$ to $-2.1 \%$ ), respectively) (paired Wilcoxon test). Both the reduction of the length and that of the total area occurred gradually during the two year treatment period (figs 2 and 3).

\section{Discussion}

This study shows that elimination of acid gastro-oesophageal reflux, as accomplished by omeprazole $40 \mathrm{mg}$ twice daily, induces regression of Barrett's epithelium, when compared with a situation of persistent pathologically increased reflux. However, the regression is small: the total area of Barrett's oesophagus was reduced by only about $8 \%$ by the end of two years treatment.

Measurement of the area and length of Barrett's oesophagus is prone to bias. The measurements are influenced by, for example, oesophageal peristalsis, the patient's breathing, the degree of flexion of the patient's neck, and the way the distance to the incisors is read in the presence of a bite block. To eliminate the variability between measurements as much as possible care was taken that at each centre one endoscopist performed the majority of the endoscopies. In addition the distances were determined while slowly withdrawing the endoscope. The necessity, according to the protocol, of making a drawing of the boundaries of the Barrett's epithelium forced the endoscopists to observe these very accurately. To reduce the impact of inherent intraobserver variations all measurements at different time 
points in one patient were combined in one parameter (the AUC) for statistical analysis; the results of this analysis corresponded to those of the simple comparisons between the data obtained at baseline and at 24 months.

The methodology for measuring the effect of therapeutic manoeuvres on Barrett's epithelium varies between different studies. Most authors report on length of Barrett's oesophagus and on the number of islands of squamous epithelium. ${ }^{10-131516}$ However, when regression occurs at the $\mathrm{Z}$ line, this regression may not be equally distributed over the whole circumference. Measurement of length may thus underestimate regression if the highest point of the $\mathrm{Z}$ line is taken as reference for the measurement, or overestimate regression if the lowest point of the $\mathrm{Z}$ line is taken as such. Counting of islands may be biased by coalescence of islands during regression, thus causing its underestimation. Furthermore, the significance of squamous islands may be limited, as a recent study showed that in about one third of islands biopsied metaplasia was still underlying the squamous epithelium. ${ }^{17}$ There is no standard method to measure the total area of Barrett's oesophagus. Weinstein et $a l^{18}$ determined area by estimating every $2 \mathrm{~cm}$ the percentage of the circumference lined with metaplastic epithelium. The most accurate method was reported by Kim et al. ${ }^{19}$ They made endoscopic photographs at 1 and $2 \mathrm{~cm}$ intervals, using videoendoscopy. By stacking these images in sequence they constructed a two dimensional map, which was used for calculating the area of Barrett's oesophagus. Our method of drawing the endoscopic findings is comparable with Kim's method but is probably less accurate. Nevertheless, in view of the acceptable intraobserver coefficients of variability and of the constancy of measurements in the ranitidine group over time, our method is apparently sufficiently reliable for the purpose of this study.

The goal of our study was to evaluate the effects of elimination of acid reflux on Barrett's oesophagus. Therefore, an unusually high dose of omeprazole was given, with apparent success, as can be concluded from the 24 hour $\mathrm{pH}$ recordings. Few of the studies reported so far have included $\mathrm{pH}$ recording to evaluate the degree of reflux reduction, but as in only one study in patients with Barrett's oesophagus such a high dose of a proton pump inhibitor was given, it is conceivable that elimination of acid reflux was not accomplished in most of those studies.

Very few studies have been reported so far that are comparable to our study. Weinstein et $a l^{18}$ reported a double blind, randomised trial comparing omeprazole $40 \mathrm{mg}$ twice daily during one year plus $20 \mathrm{mg}$ twice daily for another year with ranitidine $300 \mathrm{mg}$ twice daily. No statistically significant effects were seen in the ranitidine treated group though a trend for regression could not be excluded. The omeprazole treated group exhibited a statistically significant decrease in total area and an increase in the number of islands during the first year, without such notable changes during the second year. In another randomised trial by
Caldwell et al, comparing omeprazole $20 \mathrm{mg}$ daily with cimetidine $400 \mathrm{mg}$ three times daily for two years, neither treatment group showed a significant reduction of Barrett's oesophagus or development of squamous islands. ${ }^{14}$ No other double blind, randomised studies have been reported. Open single group studies and open controlled parallel group studies have yielded variable results with respect to reduction in length and number of squamous epithelium islands. ${ }^{10111320}$ Again, in none of these latter studies was acid inhibition so profound as in our study and as in the first year of Weinstein's study. In a well documented prospective, open, single group study, Sharma et al treated patients with lansoprazole $60 \mathrm{mg}$ per day during an average of 5.7 years. Thirteen patients underwent control $\mathrm{pH}$-metry and only eight of them had normal results with a mean $\mathrm{pH}<4$ of $0.8 \%$, whereas the other five still had pathological acid reflux despite medication. Nevertheless, symptoms improved in both groups. The authors observed no significant decrease in the length of Barrett's oesophagus, although they found a small mean decrease of $0.6 \mathrm{~cm}$ in the group with normal results at $\mathrm{pH}$-metry. In both groups were patients with a decrease as well as patients with an increase in length of Barrett's oesophagus. ${ }^{15}$ Malesci et al, on the other hand, observed a significant reduction in Barrett's oesophagus in a prospective open trial, using omeprazole 60 mg daily during 12 months. ${ }^{12}$ Of the 14 patients treated, 12 had a normalisation of their oesophageal $\mathrm{pH}$, though it did not achieve as low values as in our patients.

The regression of Barrett's oesophagus in the omeprazole treated group in our patients occurred gradually during the two year treatment period. It may thus be expected that with continued treatment the area of Barrett's oesophagus would have been reduced further. Nevertheless, the rate of redevelopment of squamous epithelium is slow and thus the time needed to accomplish complete disappearance of Barrett's oesophagus will be long in case of long stretches of Barrett's oesophagus. However, in the case of short segment Barrett's oesophagus, medical therapy may well be able to induce complete regression of Barrett's oesophagus with restitution of squamous epithelium.

Although six of the patients on ranitidine changed to open label omeprazole because of persistent reflux symptoms, a noticeable finding in this study is the paucity of symptoms in the included patients, despite high degrees of acid reflux, and the comparable improvement of symptoms during both of the two treatment modalities. The relative lack of symptoms in patients with Barrett's oesophagus has been noted before, ${ }^{21-24}$ and these symptoms can be easily alleviated with mild acid reducing treatment. A sufficient reduction or even disappearance of reflux symptoms does not automatically mean an elimination or normalisation of acid reflux. ${ }^{15}{ }^{25}$ It is therefore vital to perform a control pH-metry during medical treatment, in order to show the adequacy of acid control in a research setting. 
In conclusion, this randomised, double blind study shows that elimination of acid gastrooesophageal reflux, as accomplished by omeprazole $40 \mathrm{mg}$ twice daily, results in statistically significant regression of Barrett's oesophagus, whereas persistent pathological reflux during ranitidine $150 \mathrm{mg}$ twice daily does not. The regression is quantitatively small, however: approximately $8 \%$ in two years. Therefore, at this time, the clinical significance of this regression is to be qualified as modest and the findings do not obviate the search for other ways to induce regression. The combination of some form of coagulative ablative therapy with a high dose of a proton pump inhibitor is promising in this respect. ${ }^{26-30}$

Preliminary results of this study have been presented at the DDW/AGA 1997 in Washington DC and have been published in abstract form (Gastroenterology 1997;112:A638). Financial support was obtained from the Dutch Cancer Society, grant GUKC 91-05; the work was also supported by Astra Pharmaceutica BV, The Netherlands.

1 Paull A, Trier JS, Dalton MD, et al. The histologic spectrum of Barrett's esophagus. N Engl F Med 1976;295:476-80.

2 Spechler SJ, Robbins AH, Bloomfield Rubins H, et al. Adenocarcinoma and Barrett's esophagus. An overrated risk? Gastroenterology 1984;87:927-33.

3 Iftikhar SY, James PD, Steele RJC, et al. Length of Barrett's oesophagus: an important factor in the development of dysplasia and adenocarcinoma. Gut 1992;33:1155-8.

4 GOSPE. Barrett's esophagus and risk of adenocarcinoma [abstract]. Gastroenterology 1992;102:A75.

5 Chow W, Finkle WD, McLaughlin JK, et al. The relation of gastroesophageal reflux disease and its treatment to adenocarcinomas of the esophagus and gastric cardia. $7 A M A$ 1995;274:474-7.

6 Ritenbaugh C, Sampliner R, Aickin M, et al. Risk factors for Barrett's oesophagus: a life history approach to behavioural assessment in the distant past. Eur $f$ Cancer Prev 1995;4:459-68.

7 Menke Pluymers MBE, Hop WCJ, Dees J, et al. Risk factors for the development of an adenocarcinoma in columnarlined (Barrett) esophagus. Cancer 1993;72:1155-8.

8 Devière J, Buset M, Dumonceau J, et al. Regression of Barrett's epithelium with omeprazole. $N$ Engl f Med 1989;320: 1497-8.

9 Ottenjann R, Heidt H. Regression der ZylinderepithelMetaplasie bei Barrett-Oesophagus. Dtsch Med Wochensch 1990;115:916-17.

10 Gore S, Healey CJ, Sutton R, et al. Regression of columnar lined (Barrett's) oesophagus with continuous omeprazole therapy. Aliment Pharmacol Ther 1993;7:623-8.

11 Neumann CS, Iqbal TH, Cooper BT. Long term continuous omeprazole treatment of patients with Barrett's oesophagus. Aliment Pharmacol Ther 1995;9:451-4.

12 Malesci A, Savarino V, Zentilin P, et al. Partial regression of Barrett's esophagus by long-term therapy with high-dose omeprazole. Gastrointest Endosc 1996;44:700-5.
13 Shaffer RT, Francis J, Carrougher JG, et al. Effect of omeprazole on Barrett's epithelium after 3 years of therapy [abstract]. Gastroenterology 1996;110:A255.

14 Caldwell MTP, Byrne PJ, Walsh TN, et al. A randomised trial on the effect of acid suppression on regression of Barrett's oesophagus [abstract]. Gastroenterology 1996;110: A74.

15 Sharma P, Sampliner RE, Camargo E. Normalization of esophageal $\mathrm{pH}$ with high-dose proton pump inhibitor therapy does not result in regression of Barrett's esophagus. Am F Gastroenterol 1997;92:582-5.

16 Sampliner RE. Effect of up to 3 years of high-dose lansoprazole on Barrett's esophagus. Am f Gastroenterol 1994;89: $1844-8$.

17 Sharma P, Morales TG, Bhattacharyya A, et al. Squamous islands in Barrett's esophagus: what lies underneath? $A m \mathcal{F}$ Gastroenterol 1998;93:332-5.

18 Weinstein WM, Lieberman DA, Lewin KJ, et al. Omeprazole-induced regression of Barrett's esophagus: a 2 year, randomized, controlled double blind trial. Gastroenterology 1996;110:A294.

19 Kim R, Baggott BB, Rose S, et al. Quantitative endoscopy: precise computerized measurement of metaplastic epitheial surface area in Barrett's esophagus. Gastroenterology 1995;108:360-6.

20 Le Rhun M, Boyer J, Goldfain D, et al. Long-term omeprazole effects on Barrett's mucosa [abstract]. Gastroenterology 1996;110:A174.

21 Johnson DA, Winters C, Spurling TJ, et al. Esophageal acid sensitivity in Barrett's esophagus. F Clin Gastroenterol 1987; 9:23-7.

22 Orr WC, Lackey C, Robinson MG, et al. Esophageal acid clearance during sleep in patients with Barrett's esophagus. Dig Dis Sci 1988;33:654-9.

23 Murphy PP, Johnston BT, Collins JSA. Mucosal sensitivity and salivary response to infused acid in patients with columnar-lined oesophagus. Eur f G Gastroenterol Hepatol 1994;6:901-5.

24 Bremner RM, DeMeester TR, Bremner CG, et al. Further evidence of altered sensitivity to reflux in patients with Barrett's esophagus, Gastroenterology 1996;110:A640.

25 Katzka DA, Castell DO. Successful elimination of reflux symptoms does not insure adequate control of acid reflux in patients with Barrett's esophagus. Am $\mathcal{F}$ Gastroenterol 1994;89:989-91.

26 Berenson MM, Johnson TD, Markowitz NR, et al. Restoration of squamous mucosa after ablation of Barrett's esophageal epithelium. Gastroenterology 1993;104:168691 .

27 Sampliner RE, Fennerty MB, Garewal HS. Controlled trial of reversal of Barrett's esophagus with acid suppression and multi-polar electrocoagulation (MPEC) [abstract]. Gastroenterology 1995;108:A208.

28 Barr H, Shepherd NA, Dix A, et al. Eradication of high-grade dysplasia in columnar-lined (Barrett's) oesophagus by photodynamic therapy with endogenously generated protoporphyrin IX. Lancet 1996;348:584-5.

29 Overholt BF, Panjehpour M. Photodynamic therapy for Barrett's esophagus: clinical update. Am f Gastroenterol 1996;91:1719-23.

30 Barham CP, Jones RL, Biddlestone LR, et al. Photothermal laser ablation of Barrett's oesophagus: endoscopic and histological evidence of squamous re-epithelialisation. Gut 1997;41:281-4. 